\title{
A numerically reliable approach for the synthesis of periodic FDI filters
}

\author{
Andreas Varga \\ German Aerospace Center, DLR-Oberpfaffenhofen \\ Institute of Robotics and Mechatronics, D-82234 Wessling, Germany \\ (e-mail: andreas.varga@dlr.de)
}

\begin{abstract}
We propose an integrated numerically reliable computational approach to solve fault detection and isolation (FDI) problems for periodic systems. For this purpose, a bank of periodic fault detection filters providing a structured residual set is designed using a new least order synthesis technique of periodic fault detection filters. The synthesis procedure relies on a recently developed numerically stable algorithm to compute least order annihilators of periodic systems. This approach is extended with two additional steps for the synthesis of least order filters by using periodic dynamic cover techniques and periodic stable coprime factorizations to achieve a desired dynamics of the filters. The proposed integrated synthesis algorithm has strongly coupled computational steps, where the available structural information at the end of each step is fully exploited in the subsequent computations. All computations are done directly on the original problem data, without explicitly building time-invariant lifted system representations.
\end{abstract}

Keywords: Fault detection and isolation, periodic system, computational methods.

\section{INTRODUCTION}

The solution of the periodic fault detection and isolation problem (PFDIP) has its main application in solving fault isolation problems for multirate systems. As in the case of standard systems, the PFDIP can be solved by determining a bank of fault detection filters which provide a structured residual set.

The fault detection problem for linear multirate sampleddata systems has been considered by Fadali and EmaraShabaik (2002); Zhang et al. (2002). The proposed solutions rely on explicitly building time-invariant lifted representations of the underlying multirate systems and employing design techniques developed for standard linear systems. Although such an approach can be easily extended to the general periodic case, still there are several difficulties which can impede his usage for systems with high orders or large periods. For example, building a lifted representation using the lifting technique of (Meyer and Burrus, 1975) involves explicitly forming many matrix products, thus this approach is completely unappropriate from numerical point of view. On the other hand, using the lifting technique proposed in (Grasselli and Longhi, 1991) requires manipulating large sparse matrices of a descriptor system representation, which leads to computationally unacceptable costs. Even the final step of turning the designed lifted representation of the detector into a periodic state space representation (e.g., by using the algorithm of (Varga, 2004c)) can lead to numerical difficulties in the case of high order systems. Typical difficulties when using the lifting-based approach in conjunction with an observerbased synthesis are discussed in Zhang et al. (2012).

Recently, the fault detection problem has been considered for periodic discrete-time systems (Zhang and Ding, 2007) and a solution approach has been proposed using a periodic parity space approach. Although this approach is general and can produce fault detection filters of least possible orders, its main weakness is the need to form matrix powers and matrix products to perform rank determinations. Therefore, from a numerical point of view this method is potentially not reliable.

A lifting-free geometric approach for the synthesis of fault detection filters has been developed in (Longhi and Monteriù, 2009), using a state-space synthesis model without direct feedthrough terms. The proposed synthesis procedure determines an unknown-input observer (UIO) of the same size as the initial system. The main limitation of this approach (Chen and Patton, 1999) is the necessary condition for the existence of a stable UIO, that the system formed only with the disturbance inputs has no unstable zeros. If such zeros exist, there exists no stable UIO which can serve as a detector, although a stable fault detection filter may still exist. The approach of Longhi and Monteriù (2009) has been recently extended in (Longhi and Monteriù, 2010) to solve the fault isolation problem, by designing a bank of fault detection filters which provides a structured set of residuals. Since both in (Longhi and Monteriù, 2009) and (Longhi and Monteriù, 2010) the least order synthesis aspect has not been addressed, each of these filters has the order of the original system, and therefore the resulting global fault detection and isolation filter has potentially an unnecessary high order.

In this paper we address the fault isolation problem for periodic systems, by proposing a general approach whose main strengths lie in avoiding lifting based computations and addressing the least order synthesis aspect. The proposed approach extends to the periodic case the standard methods for constant systems to design a bank of scalar 
output filters achieving a structured residual set. The underlying method consists in solving periodic fault detection problems, using scalar output fault detection filters which are insensitive to the control and disturbance inputs as well as to a subset of faults, while being sensitive to the rest of faults. The basic computational tool is the nullspace method proposed in (Varga, 2004d), in combination with least order synthesis techniques (Varga, 2007a) and periodic coprime factorization algorithms (Varga, 2009).

Notation. For an $N$-periodic matrix $X_{k}$ we use alternativelly the script notation $\mathcal{X}:=\operatorname{diag}\left(X_{1}, X_{2}, \ldots, X_{N}\right)$, which associates the block-diagonal matrix $\mathcal{X}$ to the cyclic matrix sequence $X_{k}, k=1, \ldots, N$.

\section{PERIODIC FAULT DETECTION PROBLEMS}

We consider periodic time-varying linear discrete-time descriptor systems with additive faults of the form

$$
\begin{aligned}
E_{k} x(k+1) & =A_{k} x(k)+B_{k}^{u} u(k)+B_{k}^{d} d(k)+B_{k}^{f} f(k) \\
y(k) & =C_{k} x(k)+D_{k}^{u} u(k)+D_{k}^{d} d(k)+D_{k}^{f} f(k)
\end{aligned}
$$

where $x(k) \in \mathrm{R}^{n_{k}}$ is the system state vector with timevarying dimensions, $y(k)$ is the $p$-dimensional measurement output vector, $u(k)$ is the $m_{u}$-dimensional plant control input vector, $f(k)$ is the $m_{f}$-dimensional fault signal vector, and $d(k)$ is the $m_{d}$-dimensional disturbance vector. We assume that the system matrices are periodic with period $N \geq 1$ and $E_{k}$ are square for $k=1, \ldots, N$. For a standard periodic system, we assume $E_{k}$ is the identity matrix of appropriate size. Periodic descriptor systems will be alternatively denoted by a periodic quintuple $\left(E_{k}, A_{k}, B_{k}, C_{k}, D_{k}\right)$, while for a standard periodic system (i.e., $\left.E_{k}=I\right)$ we use only the quadruple $\left(A_{k}, B_{k}, C_{k}, D_{k}\right)$.

The periodic fault detection problem (PFDP) for linear periodic discrete-time systems can be formulated as follows: Periodic Fault Detection Problem: Determine a stable periodic linear residual generator (or detector) of the form

$$
\begin{array}{r}
\widehat{x}(k+1)=F_{k} \widehat{x}(k)+H_{k}\left[\begin{array}{l}
y(k) \\
u(k)
\end{array}\right] \\
r(k)=M_{k} \widehat{x}(k)+L_{k}\left[\begin{array}{l}
y(k) \\
u(k)
\end{array}\right]
\end{array}
$$

such that for all control inputs $u(k)$ and disturbance inputs $d(k)$ :

(i) $\|r\|_{2}=0$ if $\|f\|_{2}=0$ (fault-free case);

(ii) $\|r\|_{2} \neq 0$ if $\left\|f_{i}\right\|_{2} \neq 0$, for $i=1, \ldots, m_{f}$ (fault detectability),

where $\|\cdot\|_{2}$ is the $l_{2}$-signal norm for square summable sequences. For both $(i)$ and $(i i)$ we assume zero initial conditions for the state variables (i.e., $x(0)=0$ and $\widehat{x}(0)=$ $0)$. The stability requirement can be expressed by the condition that all characteristic multipliers of the periodic matrix $F_{k}$ (i.e., the eigenvalues of the monodromy matrix $\left.\Psi_{F}:=F_{N} \cdots F_{2} F_{1}\right)$ have moduli less than one. More generally, the stability requirement can be formulated with respect to a good region $\mathbb{C}_{g}$ of the unit disk centered in the origin, by requiring $\Lambda\left(\Psi_{F}\right) \subset \mathbb{C}_{g}$. The representation (2) is termed in (Gertler, 1998) the implementation form of the residual generator.

The so-called block fault isolation problem has been addressed by Longhi and Monteriù (2010). We consider a partition of the index set $\mathbf{I}=\left\{1,2, \ldots, m_{f}\right\}$ in $\ell$ disjoint subsets $\mathbf{I}_{i}, i=1, \ldots, \ell$ such that $\mathbf{I}=\cup_{i=1}^{\ell} \mathbf{I}_{i}$ and $\mathbf{I}_{i} \cap$ $\mathbf{I}_{j}=\emptyset$ for $i \neq j$. We are aiming to synthesize a fault detection filter which ensures that each residual signal $r_{i}(k)$ is influenced only by the corresponding faults $f_{j}(k)$ for $j \in \mathbf{I}_{i}$. The simple choice $\mathbf{I}_{i}=\{i\}$ corresponds to the standard directional residuals problem formulated in Gertler (1998).

The periodic fault detection and isolation problem (PFDIP) considered in Longhi and Monteriù (2010) can be formulated as follows:

Periodic Fault Detection and Isolation Problem: Determine a stable periodic linear residual generator with $\ell$ outputs having the general form (2) such that for all control inputs $u(k)$, all disturbance inputs $d(k)$ and for $i=1, \ldots, \ell$ :

(i) $\left\|r_{i}\right\|_{2}=0$ if $\left\|f_{j}\right\|_{2}=0 \forall j \in \mathbf{I}_{i}$;

(ii) $\left\|r_{i}\right\|_{2} \neq 0$ if $\left\|f_{j}\right\|_{2} \neq 0, \forall j \in \mathbf{I}_{i}$ (fault isolability).

For both $(i)$ and $(i i)$ we assume zero initial conditions for the state variables (i.e., $x(0)=0$ and $\widehat{x}(0)=0$ ). If the PFDIP is solvable, it is possible to detect simultaneously faults belonging to different groups defined by $\mathbf{I}_{i}, i=$ $1, \ldots, \ell$.

Both the PFDP and PFDIP can be seen as particular cases of the most general fault isolation problem using a structured set of residual. Let $S$ be a $q \times m_{f}$ structure matrix with only 0 and 1 entries, which describes the desired dependence of residuals of the individual faults as follows: the $(i, j)$-entry $S_{i j}=0$ if the $i$-th residual $r_{i}$ is not influenced by (decoupled from) the $j$-th fault input $f_{j}$, and $S_{i j}=1$ otherwise. Following the terminology of Gertler (1998), we refer to the $i$-th row of $S$ as the $i$ th specification, while the $j$-th column of $S$ is called the signature (or code) of the $j$-th fault. For example, the structure matrix $S$ corresponding to the PFDP is a $1 \times m_{f}$ row vector with entries only ones

$$
S=\left[\begin{array}{llll}
1 & 1 & \cdots & 1
\end{array}\right]
$$

while for the PFDIP $S$ has a block-diagonal form, where the diagonal blocks are row vectors of appropriate sizes with entries only ones. Generally, a structured residual set can be employed to perform fault isolation under weaker conditions. For example, if it can be assumed that two faults never occur simultaneously, then the simple structure (e.g., for three faults)

$$
S=\left[\begin{array}{lll}
0 & 1 & 1 \\
1 & 0 & 1 \\
1 & 1 & 0
\end{array}\right]
$$

can be used to detect individual faults by comparing the fired/not fired structured residual evaluation signal with the fault signatures coded in $S$.

The periodic structured fault detection and isolation problem (PSFDIP) can be formulated as follows:

Periodic Structured Fault Detection and Isolation Problem: For a given $q \times m_{f}$ structure matrix $S$, determine a stable periodic linear residual generator with $q$ outputs having the general form (2) such that for all control inputs $u(k)$, all disturbance inputs $d(k)$ and for $i=1, \ldots, q$ :

(i) $\left\|r_{i}\right\|_{2}=0$ if $\left\|f_{j}\right\|_{2}=0 \forall j$ with $S_{i, j}=1$;

(ii) $\left\|r_{i}\right\|_{2} \neq 0$ if $\left\|f_{j}\right\|_{2} \neq 0, \forall j$ with $S_{i, j}=1$. 
For both $(i)$ and $(i i)$ we assume, as before, zero initial conditions for the state variables (i.e., $x(0)=0$ and $\widehat{x}(0)=0)$.

In this paper, we only address the solution of the PSFDIP, which, as already mentioned, encompasses also the solution of the PFDIP. As in the case of standard systems (Gertler, 1998), we can solve the PSFDIP by solving $q$ PFDPs by designing a bank of $q$ periodic fault detection filters of the form (2) with scalar outputs, such that the $i$ th filter is sensitive to faults according to the $i$-th specification of the given structure matrix $S$. More concretely, the synthesis problem for the $i$-th detector can be reformulated as a standard PFDP with redefined and permuted inputs. Let $B_{k}^{f_{\hat{\imath}}}$ and $D_{k}^{f_{\hat{\imath}}}$ be the matrices formed from the columns of matrices $B_{k}^{f}$ and $D_{k}^{f}$ with column indices $j$ with $S_{i j}=1$, respectively, and let $B_{k}^{f_{\bar{i}}}$ and $D_{k}^{f_{\bar{i}}}$ be the rest of columns in these matrices. We can redefine the matrices of the model (1) using the following replacements

$$
\begin{aligned}
& B_{k}^{d} \leftarrow \widetilde{B}_{k}^{d}:=\left[B_{k}^{d} B_{k}^{f_{\bar{i}}}\right], \quad B_{k}^{f} \leftarrow \widehat{B}_{k}^{f}:=B_{k}^{f_{\hat{i}}} \\
& D_{k}^{d} \leftarrow \widetilde{D}_{k}^{d}:=\left[D_{k}^{d} D_{k}^{f_{\bar{i}}}\right], \quad D_{k}^{f} \leftarrow \widehat{D}_{k}^{f}:=D_{k}^{f_{\hat{\imath}}}
\end{aligned}
$$

and solve a standard PFDP to determine the $i$-th filter in the form (2). Thus, the PSFDIP problem can be solved by solving $q$ standard PFDP. A similar approach has been considered in (Longhi and Monteriù, 2010) for solving the PFDIP using full order UIOs. Besides removing the already mentioned limitations of this approach, in this paper we address additionally the synthesis of fault detection filters with the least achievable order. This aspect is crucial in achieving reasonable low orders of the resulting global FDI filter obtained by stacking the $q$ individual scalar output filters.

In what follows, we will focus only on the solution of the PFDP using reliable numerical techniques. However, before addressing the numerical issues, we present in the next section the solvability conditions of the formulated problems using the lifted linear time-invariant (LTI) representations of periodic systems.

\section{SOLVABILITY CONDITIONS}

To derive algebraic conditions for the solvability of the PFDP, PFDIP and PSFDIP, we reformulate the detector design problems in terms of the transfer-function matrix (TFM) corresponding to the associated stacked lifted representation of (Grasselli and Longhi, 1991), which uses the input-state-output behavior of the system over time intervals of length $N$, rather then 1. The lifted input, output and state vectors are defined as

$$
\begin{aligned}
& \widetilde{u}(h)=\left[u^{T}(h N+1) \cdots u^{T}(h N+N)\right]^{T}, \\
& \widetilde{d}(h)=\left[d^{T}(h N+1) \cdots d^{T}(h N+N)\right]^{T}, \\
& \widetilde{f}(h)=\left[f^{T}(h N+1) \cdots f^{T}(h N+N)\right]^{T}, \\
& \widetilde{y}(h)=\left[y^{T}(h N+1) \cdots y^{T}(h N+N)\right]^{T}, \\
& \widetilde{x}(h)=\left[x^{T}(h N+1) \cdots x^{T}(h N+N)\right]^{T} .
\end{aligned}
$$

and the corresponding lifted system can be represented by a LTI descriptor system of the form (notice the usage of script notation)

$$
\begin{aligned}
\widetilde{E} \widetilde{x}(h+1) & =\widetilde{A} \widetilde{x}(h)+\mathcal{B}^{u} \widetilde{u}(h)+\mathcal{B}^{d} \widetilde{d}(h)+\mathcal{B}^{f} \widetilde{f}(h) \\
\widetilde{y}(h) & =\mathcal{C} \widetilde{x}(h)+\mathcal{D}^{u} \widetilde{u}(h)+\mathcal{D}^{d} \widetilde{d}(h)+\mathcal{D}^{f} \widetilde{f}(h)
\end{aligned}
$$

where the pole pencil corresponding to the periodic pair $\left(A_{k}, E_{k}\right)$

$$
\widetilde{A}-z \widetilde{E}=\left[\begin{array}{ccccc}
A_{1} & -E_{1} & 0 & \cdots & 0 \\
0 & \ddots & \ddots & \ddots & \vdots \\
\vdots & \ddots & \ddots & -E_{N-2} & 0 \\
0 & & \ddots & A_{N-1} & -E_{N-1} \\
-z E_{N} & 0 & \cdots & 0 & A_{N}
\end{array}\right]
$$

is assumed regular. For the lifted system, the TFMs $G_{u}(z)$, $G_{d}(z), G_{f}(z)$ from the control, disturbance, and fault inputs, respectively, to the system output are

$$
G_{\xi}(z)=\mathcal{C}(z \widetilde{E}-\widetilde{A})^{-1} \mathcal{B}^{\xi}+\mathcal{D}^{\xi}
$$

where $\xi$ stays for $u, d$ or $f$. Let denote by $G_{f_{i}}(z)$ the TFM of the lifted system corresponding to the $i$-th fault defined as

$$
G_{f_{i}}(z)=\mathcal{C}(z \widetilde{E}-\widetilde{A})^{-1} \mathcal{B}^{f_{i}}+\mathcal{D}^{f_{i}}
$$

where $B_{k}^{f_{i}}$ and $D_{k}^{f_{i}}$ are the $i$-th columns of matrices $B_{k}^{f}$ and $D_{k}^{f}$, respectively.

Assume that the linear residual generator (2) has a lifted representation with the corresponding TFM $Q(z)$. Let $R_{u}(z), R_{d}(z)$ and $R_{f}(z)$ be the corresponding TFMs from the control, disturbance and fault inputs to the residual. These TFMs can be expressed as

$$
\left[R_{u}(z) R_{d}(z) R_{f}(z)\right]=Q(z)\left[\begin{array}{ccc}
G_{u}(z) & G_{d}(z) & G_{f}(z) \\
I_{N m_{u}} & 0 & 0
\end{array}\right]
$$

The periodic realization corresponding to the above lifted TFMs in the left hand side is termed in (Gertler, 1998) the internal form of the residual generator.

For the PFDP, the condition $(i)$ requires $R_{u}(z)=0$ and $R_{d}(z)=0$, or equivalently

$$
Q(z) G(z)=0
$$

where

$$
G(z)=\left[\begin{array}{cc}
G_{u}(z) & G_{d}(z) \\
I_{N m_{u}} & 0
\end{array}\right]
$$

If we denote by $R_{f_{i}}(z)$ the TFM of the lifted system corresponding to the $i$-th fault, the fault detectability condition $(i i)$ requires $R_{f_{i}}(z) \neq 0$, for $i=1, \ldots, m_{f}$, or equivalently

$$
Q(z)\left[\begin{array}{c}
G_{f_{i}}(z) \\
0
\end{array}\right] \neq 0, \quad i=1, \ldots, m_{f}
$$

The synthesis conditions (7) and (9) must be complemented with the requirements of stability for $Q(z)$ and $R_{f}(z)$, as well as the least possible order of $Q(z)$.

The necessary and sufficient condition for the existence of a solution to the PFDF is the following one (Ding and Frank, 1991; Nyberg, 2002):

Theorem 1. For the periodic system (1) the PFDP is solvable if and only if

$$
\operatorname{rank}\left[G_{d}(z) G_{f_{i}}(z)\right]>\operatorname{rank} G_{d}(z), i=1, \ldots, m_{f}
$$

Since the PFDIP can be seen as a particular PSFDIP, we give the solvability conditions only for the latter case. These conditions are simply the cumulative solvability conditions for the existence of $q$ filters, where the $i$ th filter must achieve the $i$-th specification contained in the $i$-th row of $S$. Let $\widetilde{G}_{d}^{(i)}(z)$ and $\widehat{G}_{f}^{(i)}(z)$ be the lifted 
TFMs corresponding to the redefined disturbance and fault inputs using the replacements in (3). A necessary and sufficient condition for the existence of a solution to the PSFDIP is:

Theorem 2. For the periodic system (1) the PSFDIP is solvable for a given $S$ if and only if for $i=1, \ldots, q$

$$
\operatorname{rank}\left[\widetilde{G}_{d}^{(i)}(z) \widehat{G}_{f}^{(i)}(z)\right]>\operatorname{rank} \widetilde{G}_{d}^{(i)}(z)
$$

A synthesis procedure of a residual generation filter which solves the PFDP, can be also employed to solve the PFDIP or PSFDIP. This can be done by constructing explicitly the lifted representations and applying methods developed for time-invariant descriptor systems (see for example (Varga, 2003a, 2007b)). Since resulting $Q(z)$ for the PFDP must be a left annihilator of $G(z)$, one possibility to determine $Q(z)$ is to compute first a left minimal proper rational basis $N(z)$ for the left nullspace of $G(z)$, and then to build a rational and stable detector as $Q(z)=X(z) N(z)$, where $X(z)$ is chosen such that $Q(z)$ is stable, the detector fulfills the fault detectability condition $(i i)$, and has least order. To ensure stability, periodic left coprime factorization techniques can employed. The main difficulty with such an approach is that we must ensure that the resulting $Q(z)$ corresponds to a lifted causal periodic system which must be realizable in the form (2). Therefore, constructing $N(z)$ and choosing $X(z)$ must correspond to causal periodic realizations, with the additional constraint that the conditions (9) are fulfilled. This appears to be far from a trivial computational task. Moreover, even in the case when this approach is applicable, severe numerical difficulties are to be expected for systems with large periods and/or orders.

In the next sections, we show that an equivalent approach is possible for periodic systems without resorting to manipulate explicitly lifted representations. The proposed computational approach operates directly on the matrices of the original periodic state-space description (1) and computes left annihilators directly in minimal periodic state-space form. All subsequent computations to determine a least order stable detector which satisfies the fault detectability constraints are performed on this representation and can be done using reliable numerical techniques based on state-space computations as well.

\section{SOLVING THE LEAST ORDER PFDP}

The three main steps of solving the PFDP follows the approach of Varga (2003b) for standard systems: (1) computation of a maximal left annihilator; (2) computation of a least order detector, and (3) assigning a desired dynamics for the detector. In what follows we describe these steps and indicate suitable algorithms to be used.

\subsection{Computation of a maximal left annihilator}

In this section we describe a computational approach to determine a maximal left annihilator for the periodic system

$$
\begin{aligned}
E_{k} x(k+1) & =A_{k} x(k)+B_{k}^{u} u(k)+B_{k}^{d} d(k) \\
{\left[\begin{array}{c}
y(k) \\
u(k)
\end{array}\right] } & =\left[\begin{array}{c}
C_{k} \\
0
\end{array}\right] x(k)+\left[\begin{array}{c}
D_{k}^{u} \\
I_{m_{u}}
\end{array}\right] u(k)+\left[\begin{array}{c}
D_{k}^{d} \\
0
\end{array}\right] d(k)
\end{aligned}
$$

which corresponds to the lifted TFM $G(z)$ in (8). In terms of lifted representations, this amounts to determine a periodic system (e.g., of the form (2)), whose lifted TFM $N(z)$ is a proper rational matrix whose columns represent a basis for the left nullspace of $G(z)$ (i.e., $N(z) G(z)=0$ ).

A suitable computational approach is described in detail in (Varga, 2004d) and therefore we give here only the essential computations. Consider the periodic system matrix pair $\left(S_{k}, T_{k}\right)$ defined as

$$
S_{k}=\left[\begin{array}{ccc}
A_{k} & B_{k}^{u} & B_{k}^{d} \\
C_{k} & D_{k}^{u} & D_{k}^{d} \\
0 & I_{m_{u}} & 0
\end{array}\right], \quad T_{k}=\left[\begin{array}{ccc}
E_{k} & 0 & 0 \\
0 & 0 & 0 \\
0 & 0 & 0
\end{array}\right]
$$

Let $Q_{k}$ and $Z_{k}$ be orthogonal $N$-periodic matrices determined using the algorithm proposed in (Varga, 2004b) to reduce the $N$-periodic pair $\left(S_{k}, T_{k}\right)$ to the Kronecker-like form $\left(\bar{S}_{k}, \bar{T}_{k}\right):=\left(Q_{k} S_{k} Z_{k}, Q_{k} T_{k} Z_{k+1}\right)$, where

$$
\bar{S}_{k}=\left[\begin{array}{c|ccc}
B_{k}^{r} & A_{k}^{r} & * & * \\
0 & 0 & A_{k}^{r e g} & * \\
0 & 0 & 0 & A_{k}^{l} \\
\hline 0 & 0 & 0 & C_{k}^{l}
\end{array}\right], \quad \bar{T}_{k}=\left[\begin{array}{c|ccc}
0 & E_{k}^{r} & * & * \\
0 & 0 & E_{k}^{r e g} & * \\
0 & 0 & 0 & E_{k}^{l} \\
\hline 0 & 0 & 0 & 0
\end{array}\right]
$$

where: $(a)$ the periodic system $\left(E_{k}^{r}, A_{k}^{r}, B_{k}^{r}, *, *\right)$ is completely reachable and $E_{k}^{r}$ is invertible; $(b)$ the periodic system $\left(E_{k}^{l}, A_{k}^{l}, *, C_{k}^{l}, *\right)$ is completely observable and $E_{k}^{l}$ is invertible; $(c)$ the pole pencil (5) corresponding to the periodic pair $\left(E_{k}^{r e g}, A_{k}^{r e g}\right)$ is regular. Note that the triples $\left(E_{k}^{r}, A_{k}^{r}, B_{k}^{r}\right)$ and $\left(E_{k}^{l}, A_{k}^{l}, C_{k}^{l}\right)$ specify the right and left Kronecker structures of the system matrix $S(z)$ corresponding to $G(z)$, respectively, while the pair $\left(E_{k}^{r e g}, A_{k}^{r e g}\right)$ specifies the finite and infinite zero structure of $S(z)$.

With the resulting periodic orthogonal matrix $Q_{k}$, we also compute

$$
Q_{k}\left[\begin{array}{cc|c}
0 & 0 & B_{k}^{f} \\
I_{p} & 0 & D_{k}^{f} \\
0 & I_{m_{u}} & 0
\end{array}\right]=\left[\begin{array}{c|c}
\widehat{B}_{k} & * \\
B_{k}^{l} & \widetilde{B}_{k}^{f} \\
D_{k}^{l} & \widetilde{D}_{k}^{f}
\end{array}\right]
$$

and build the periodic realizations for $N(z)$ of the form

$$
\begin{aligned}
E_{k}^{l} \bar{x}(k+1) & =A_{k}^{l} \bar{x}(k)+B_{k}^{l}\left[\begin{array}{c}
y(k) \\
u(k)
\end{array}\right] \\
\bar{y}(k) & =C_{k}^{l} \bar{x}(k)+D_{k}^{l}\left[\begin{array}{c}
y(k) \\
u(k)
\end{array}\right]
\end{aligned}
$$

and of

in the form

$$
N_{f}(z):=N(z)\left[\begin{array}{c}
G_{f}(z) \\
0
\end{array}\right]
$$

$$
\begin{aligned}
E_{k}^{l} x_{f}(k+1) & =A_{k}^{l} x_{f}(k)+\widetilde{B}_{k}^{f} f(k) \\
r(k) & =C_{k}^{l} x_{f}(k)+\widetilde{D}_{k}^{f} f(k)
\end{aligned}
$$

As it can be observed, the two realizations of $N(z)$ and $N_{f}(z)$ share the matrices $E_{k}^{l}, A_{k}^{l}$, and $C_{k}^{l}$. Since $E_{k}^{l}$ is nonsingular, these periodic descriptor systems can be easily reduced to standard ones as in (2).

Taking into account that the realization (16) is observable, the condition (10) for checking the solvability of the PFDP can be expressed as (Varga, 2004d, Theorem 3):

$$
\exists k \text { such that }\left[\begin{array}{c}
\widetilde{B}_{k}^{f_{i}} \\
\widetilde{D}_{k}^{f_{i}}
\end{array}\right] \neq 0, \quad i=1, \ldots, m_{f}
$$

It is important to emphasize that for the determination of the left annihilator (14), a single reduction of a periodic pair to a periodic Kronecker-like form has been 
performed using exclusively orthogonal transformations on the pairs of matrices $\left(S_{k}, T_{k}\right)$. It is possible to prove that all computed matrices are exact for a slightly perturbed original system matrix pairs. It follows that the algorithm to compute the left annihilator (14) and the corresponding realization of $N_{f}(z)$ in (16) is numerically stable.

\subsection{Least order synthesis using dynamic covers}

Without loosing from the generality, we can assume in what follows that each $E_{k}^{l}$ in (14) and (16) is the identity matrix of the corresponding size. This can be achieved by replacing in (14) $A_{k}^{l}$ by $\left(E_{k}^{l}\right)^{-1} A_{k}^{l}$ and $B_{k}^{l}$ by $\left(E_{k}^{l}\right)^{-1} B_{k}^{l}$, and similarly, replacing in (16) $A_{k}^{l}$ by $\left(E_{k}^{l}\right)^{-1} A_{k}^{l}$ and $\widetilde{B}_{k}^{f}$ by $\left(E_{k}^{l}\right)^{-1} \widetilde{B}_{k}^{f}$.

Let $H_{k}$ and $K_{k}$ periodic matrices and consider a periodic prefilter with the lifted TFM $W(z)$ and the standard periodic realization $\left(A_{k}^{l}+K_{k} C_{k}^{l}, K_{k}, H_{k} C_{k}^{l}, H_{k}\right)$. It is straightforward to show, that the lifted TFM $W(z) N(z)$ has the periodic realization

$$
\left(A_{k}^{l}+K_{k} C_{k}^{l}, B_{k}^{l}+K_{k} D_{k}^{l}, H_{k} C_{k}^{l}, H_{k} D_{k}^{l}\right)
$$

In this section we address the problem to determine for a given periodic $H_{k}$, the periodic $K_{k}$ which makes the above realization maximally unobservable.

The computation of such a $K_{k}$ can pe performed using recently developed minimal dynamic cover techniques for standard periodic systems Varga (2007a). The general idea of the cover algorithms is to perform a preliminary orthogonal similarity transformation on the periodic system matrices in (14) by applying a special version of the periodic controllability staircase form algorithm of Varga (2004a) to the dual periodic descriptor pair $\left(\left(A_{N+1-k}^{l}\right)^{T},\left[\left(H_{N+1-k} C_{N+1-k}^{l}\right)^{T}\left(C_{N+1-k}^{l}\right)^{T}\right]\right)$ and then with additional block permutations and nonorthogonal block row/column transformations, the transformed system matrices are put in a special form which allows to cancel the maximum number of poles. For the so-called Type I dynamic covers, a nonsingular periodic transformation matrix $U_{k}$ results such that

$$
\begin{gathered}
U_{k+1}^{-1} A_{k}^{l} U_{k}=\left[\begin{array}{c|c}
\widehat{A}_{k, 11} & \widehat{A}_{k, 12} \\
\hline \widehat{A}_{k, 21} & \widehat{A}_{k, 22}
\end{array}\right], \\
U_{k+1}^{-1} B_{k}^{l}=\left[\frac{\widehat{B}_{k, 1}}{\widehat{B}_{k, 2}}\right], \quad\left[\begin{array}{c}
C_{k}^{l} \\
\hline H_{k} C_{k}^{l}
\end{array}\right] U_{k}=\left[\begin{array}{c|c}
\widehat{C}_{k, 11} & \widehat{C}_{k, 12} \\
\hline 0 & \widehat{C}_{k, 22}
\end{array}\right],
\end{gathered}
$$

where the periodic pairs $\left(\widehat{A}_{k, 11}, \widehat{C}_{k, 11}\right)$ and $\left(\widehat{A}_{k, 22}, \widehat{C}_{k, 22}\right)$ are observable, and the submatrices $\widehat{C}_{k, 11}$ and $\widehat{A}_{k, 21}$ have the particular structure

$$
\left[\begin{array}{c}
\widehat{A}_{k, 21} \\
\widehat{C}_{k, 11}
\end{array}\right]=\left[\begin{array}{ll}
0 & A_{k, 21} \\
0 & C_{k, 11}
\end{array}\right]
$$

with $C_{k, 11}$ having full column rank. By taking

$$
K_{k}=U_{k+1}^{-1}\left[\begin{array}{c}
0 \\
K_{k, 2}
\end{array}\right]
$$

with $K_{k, 2}$ satisfying $K_{k, 2} C_{k, 11}+A_{k, 21}=0$, we annihilate $\widehat{A}_{k, 21}$, and thus make the part containing $\widehat{A}_{k, 11}$ unobservable. The resulting realization of $W(z) N(z)$ of least McMillan degree is obtained by deleting the unobservable part and thus has the minimal state space realization

$$
\left(\widehat{A}_{k, 22}+K_{k, 2} \widehat{C}_{k, 12}, \widehat{B}_{k, 2}+K_{k, 2} D_{k}^{l}, \widehat{C}_{k, 22}, H_{k} D_{k}^{l}\right)
$$

The periodic realization of $W(z) N_{f}(z)$ can be computed in the form

$$
\left(\widehat{A}_{k, 22}+K_{k, 2} \widehat{C}_{k, 12}, \widehat{B}_{k, 2}^{f}+K_{k, 2} \widetilde{D}_{k}^{f}, \widehat{C}_{k, 22}, H_{k} \widetilde{D}_{k}^{f}\right)
$$

where

$$
U_{k+1}^{-1} \widetilde{B}_{k}^{f}=\left[\frac{\widehat{B}_{k, 1}^{f}}{\widehat{B}_{k, 2}^{f}}\right]
$$

The choice of suitable $H_{k}$ is crucial for the success of this approach. This choice is directed, similarly as explained for the standard case in Varga (2007b), by the fine structure of the periodic pair $\left(A_{k}^{l}, C_{k}^{l}\right)$ which is in a periodic observability staircase form. For example, employing a vector $H_{k}$ with $j$ nonzero components in the first positions is equivalent to forming a linear combination of $j$ annihilators which corresponds to the nonzero components of $H_{k}$. Starting with a single component and incresing successively the number of non-zero components produces annihilators of increasing orders. The process stops when the following fault detectability conditions for the updated realization of $W(z) N_{f}(z)$ are fulfilled:

$$
\forall i \exists k \text { such that }\left[\begin{array}{c}
\widehat{B}_{k, 2}^{f_{i}}+K_{k, 2} \widetilde{D}_{k}^{f_{i}} \\
H_{k} \widetilde{D}_{k}^{f_{i}}
\end{array}\right] \neq 0
$$

The computation of the transformation matrix $U_{k}$ involves two stages. First, an orthogonal reduction involving rank decisions is performed. This computation is numerically stable. In a second phase, zero entries are successively created in the transformed state matrices using nonorthogonal transformations with a special block-triangular structure. The condition numbers of the transformation matrices can be easily determined (being related to the norm of the off-diagonal part), and unstable computations can be easily detected if these norms exceed a certain threshold. In this way, the cover algorithm can be considered a reliable algorithm, although it is not numerically stable. Moreover, the explicit computation of $U_{k}$ is not necessary if the transformation are also applied to the input matrix $\widetilde{B}_{k}^{f}$ of the realization of $N_{f}(z)$ in (16).

Note: By employing a straightforward extension of the periodic cover algorithm of (Varga, 2007a) to periodic descriptor systems, the inversion of $E_{k}^{l}$ can be also avoided.

\subsection{Stabilization via periodic coprime factorization}

To simplify the notations, assume that $W(z) N(z)$ and $W(z) N_{f}(z)$ have the periodic realizations $\left(\bar{A}_{k}^{l}, \bar{B}_{k}^{l}, \bar{C}_{k}^{l}, \bar{D}_{k}^{l}\right)$ and $\left(\bar{A}_{k}^{l}, \bar{B}_{k}^{f}, \bar{C}_{k}^{l}, \bar{D}_{k}^{f}\right)$, respectively. Let $\mathbb{C}_{g}$ be the desired stability region for the characteristic multipliers of the fault detection filter. If $\Lambda\left(\Psi_{\bar{A}^{l}}\right) \not \subset \mathbb{C}_{g}$, then a stable left coprime factorization can be computed in the form

$$
\left[W(z) N(z) W(z) N_{f}(z)\right]=M^{-1}(z)\left[Q(z) R_{f}(z)\right]
$$

where all intervening TFMs in the right hand side have only poles in $\mathbb{C}_{g}$. For this, the periodic realization of $M(z)$ is chosen in the form $\left(\bar{A}_{k}^{l}+\bar{K}_{k} \bar{C}_{k}^{l}, \bar{K}_{k}, \bar{C}_{k}^{l}, I\right)$ and the corresponding $Q(z)$ and $R_{f}(z)$ have the periodic realizations $\left(\bar{A}_{k}^{l}+\bar{K}_{k} \bar{C}_{k}^{l}, \bar{B}_{k}^{l}+\bar{K}_{k} \bar{D}_{k}^{l}, \bar{C}_{k}^{l}, \bar{D}_{k}^{l}\right)$ and respectively $\left(\bar{A}_{k}^{l}+\bar{K}_{k} \bar{C}_{k}^{l}, \bar{B}_{f}+\bar{K}_{k} \bar{D}_{k}^{f}, \bar{C}_{k}^{l}, \bar{D}_{k}^{f}\right)$. The periodic matrix $\bar{K}_{k}$ can be determined using the numerically reliable periodic coprime factorization algorithm of (Varga, 2009). 


\section{CONCLUSIONS}

The numerical solution of the PFDP (and also of the related PFDIP and PSFDIP) can be performed using a numerically reliable integrated computational algorithm, where the three successive computational steps are closely connected. At the end of each computational step, we have a candidate residual generator which can be even deployed, provided the stability requirement is fulfilled. Both the implementation and internal forms of the residual generator have the same state dynamics and observation matrices at the end of each computational step. Thus, the proposed synthesis procedure can be interpreted as a successive updating of detector representations. This leads to a substantial flexibility in performing the detector synthesis, allowing for example, the skipping of the least order synthesis step or performing the stabilization step, only if it is necessary.

The least order synthesis step fully exploits the staircase structure of the periodic pairs $\left(A_{k}^{l}, C_{k}^{l}\right)$ in choosing appropriate linear combinations via the matrices $H_{k}$. Also, the triangular shape of the matrix $E_{k}^{l}$ can be exploited in the updating of matrices of the computed left annihilator as described in subsection 4.2. This confers an even more integrated character of the computational steps.

When solving the PFDIP or PSFDIP, it is possible to perform a preliminary preprocessing step (as described in subsection 4.1) to decouple all control inputs and disturbances via a maximal left annihilator. In this way, the original PFDIP or PSFDIP can be replaced by simpler problems without control and disturbance inputs. The new synthesis model is in fact the resulting internal form of the residual generator, for which a bank of detectors can be now designed using the described three-steps procedure. Once again, the described updating techniques can be employed to determine the least order representations for each of the $q$ detectors. Such enhancements for standard systems are discussed in (Varga, 2011).

Several connected problems are worth to be investigated in the future, as for example the determination of the achievable structure matrix for a given periodic system, or the extension of the PFDP to address the approximate solution in the case when additional noise inputs are present. In this context, an algorithm to compute innerouter factorizations of periodic systems needs to be still developed.

\section{REFERENCES}

Chen, J. and Patton, R.J. (1999). Robust Model-Based Fault Diagnosis for Dynamic Systems. Kluwer Academic Publishers, London.

Ding, X. and Frank, P.M. (1991). Frequency domain approach and threshold selector for robust model-based fault detection and isolation. Proc. of IFAC Symposium SAFEPROCESS'1991, Baden-Baden, Germany.

Fadali, M.S. and Emara-Shabaik, H.E. (2002). Timely robust fault detection for multirate linear systems. Int. J. Control, 75, 305-313.
Gertler, J. (1998). Fault Detection and Diagnosis in Engineering Systems. Marcel Dekker, New York.

Grasselli, O.M. and Longhi, S. (1991). Finite zero structure of linear periodic discrete-time systems. Int. J. Systems Sci., 22, 1785-1806.

Longhi, S. and Monteriù, A. (2009). Fault detection for linear periodic systems using a geometric approach. IEEE Trans. Automat. Control, 54, 1637-1643.

Longhi, S. and Monteriù, A. (2010). A geometric approach to fault detection and isolation problem for linear periodic systems. Proc. of CDC'10, Atlanta, USA.

Meyer, R.A. and Burrus, C.S. (1975). A unified analysis of multirate and periodically time-varying digital filters. IEEE Trans. Circuits Syst., 22, 162-168.

Nyberg, M. (2002). Criterions for detectability and strong detectability of faults in linear systems. Int. J. Control, $75,490-501$.

Varga, A. (2003a). On computing least order fault detectors using rational nullspace bases. Proc. of IFAC Symp. SAFEPROCESS'2003, Washington D.C.

Varga, A. (2003b). Reliable algorithms for computing minimal dynamic covers. Proc. of CDC'2003, Maui, Hawaii.

Varga, A. (2004a). Computation of Kalman decompositions of periodic systems. European Journal of Control, $10,1-8$

Varga, A. (2004b). Computation of Kronecker-like forms of periodic matrix pairs. Proc. of MTNS'04, Leuven, Belgium.

Varga, A. (2004c). Computation of minimal periodic realizations of transfer-function matrices. IEEE Trans. Automat. Control, 46, 146-149.

Varga, A. (2004d). Design of fault detection filters for periodic systems. Proc. of $C D C^{\prime} 04$, Paradise Island, Bahamas.

Varga, A. (2007a). On computing minimal dynamic covers for periodic systems. Proc. of ECC'07, Kos, Greece.

Varga, A. (2007b). On designing least order residual generators for fault detection and isolation. Proc. 16th Internat. Conf. on Control Systems and Computer Science, Bucharest, Romania, 323-330.

Varga, A. (2009). On computing periodic coprime factorizations. Proc. ECC'2009, Budapest, Hungary.

Varga, A. (2011). On computing minimal proper nullspace bases with applications in fault detection. In P.V. Dooren, S.P. Bhattacharyya, R.H. Chan, V. Olshevsky, and A. Routray (eds.), Numerical Linear Algebra in Signals, Systems and Control, vol. 80 of Lecture Notes in Electrical Engineering, 433-465. Springer Verlag, Berlin.

Zhang, P. and Ding, S.X. (2007). Disturbance decoupling in fault detection of linear periodic systems. Automatica, 43, 1410-1417.

Zhang, P., Ding, S.X., and Liu, P. (2012). A lifting based approach to observer based fault detection of linear periodic systems. IEEE Trans. Automat. Control, 57, 457-462.

Zhang, P., Ding, S.X., Wang, G.Z. and Zhou, D.Z. (2002). Fault detection for multirate sampled-data systems with time delay. Int. J. Control, 75, 1457-1471. 